\title{
Respiratory syncytial virus-related encephalitis: magnetic resonance imaging findings with diffusion-weighted study: response to a "letter to the editor"
}

\author{
Arim Park • Sangil Suh
}

Received: 10 February 2014 / Accepted: 12 February 2014 / Published online: 27 February 2014

(C) Springer-Verlag Berlin Heidelberg 2014

\section{Dear Sir,}

We thank Dr. Donmez for his comments on our publication in Neuroradiology [1], and we would like to make the following comments in reply.

He noted that the normal diffusion-weighted imaging (DWI) of our cases may not be associated with a good prognosis if brain magnetic resonance imaging (MRI) with diffusion study was performed in the subacute phase of cytotoxic edema, in which restricted diffusion start to diminish and apparent diffusion coefficient (ADC) values increase. We agree with him that timing is important in brain MRI with diffusion study in patients with encephalitis, and DWI may be less sensitive when there is a time delay between the onset of the disease and imaging.

In all three of our cases, the pediatric physicians decided to perform DWI within the first $12 \mathrm{~h}$ and conventional brain MRI within the first 3 days immediately after meaningful neurologic symptom onset. There was no definite restriction on the diffusion study including ADC map in our cases. So we could not find the evidence of cytotoxic edema induced by encephalitis at acute phase in our cases by using diffusion study [2]. As we expected, these patients showed favorable outcome with supportive management.

In a previous report, enterovirus 71-related encephalomyelitis with brain MRI and DWI study, we experienced that four of five patients did not reveal definite restriction on the diffu-

This letter is a response to an original letter to the editor "Respiratory syncytial virus-related encephalitis: magnetic resonance imaging findings with diffusion-weighted study," DOI 10.1007/s00234-014-1337-z.

\section{A. Park $\cdot$ S. Suh $(\bowtie)$}

Department of Radiology, Korea University Guro Hospital,

Korea University School of Medicine, 97 Gurodong, Guro-gu,

Seoul 152-703, South Korea

e-mail:ssickh@korea.ac.kr sion study, who gradually recovered with conservative treatment without sequelae. But, one case with restricted diffusion in the lesion of the hippocampus showed the atrophic change on follow-up study [3].

Consequently, we consider that no definite restriction on the diffusion study in the early phase of the RSV-related encephalitis may provide evidence for the reversibility of the lesion, which is not progressed to cytotoxic edema and it is associated with favorable outcomes $[4,5]$.

As Dr. Donmez commented, DWI is superior to conventional MRI in detecting irreversible cytotoxic edema especially at acute phase of encephalitis. However, it may be less sensitive at later phase due to dynamic pathologic changes. It is needed to be considered and discussed at further study.

Conflict of interest We declare that we have no conflict of interest.

\section{References}

1. Park A, Suh SI, Son GR, Lee YH, Seo HS, Eun BL, Seol HY (2014) Respiratory syncytial virus-related encephalitis: magnetic resonance imaging findings with diffusion-weighted study. Neuroradiology 56(2):163-168

2. Kiroğlu Y, Calli C, Yunten N, Kitis O, Kocaman A, Karabulut N, Isaev H, Yagci B (2006) Diffusion-weighted MR imaging of viral encephalitis. Neuroradiology 48(12):875-880

3. Jang S, Suh SI, Ha SM, Byeon JH, Eun BL, Lee YH, Seol HY (2012) Enterovirus 71-related encephalomyelitis: usual and unusual magnetic resonance imaging findings. Neuroradiology 54(3):239-245

4. Kim JH, Joo BE, Koh SB (2007) Serial diffusion-weighted MR imaging findings in a patient with Epstein-Barr virus encephalitis. $\mathrm{J}$ Neurol 254:1616-1618

5. Schaefer PW, Grant PE, Gonzalez RG (2000) Diffusion-weighted MR imaging of the brain. Radiology 217:331-345 